\title{
Fragmented QRS and other depolarization abnormalities in Nigerians with heart failure: Prevalence and clinical correlates
}

\author{
Adeseye A Akintunde MBChB FWACP FMCP FACC FESC ${ }^{1,2}$
}

AA Akintunde. Fragmented QRS and other depolarization abnormalities in Nigerians with heart failure: Prevalence and clinical correlates. Curr Res Cardiol 2015;2(3):105-108.

BACKGROUND: Fragmented QRS (fQRS), QT prolongation and wide QRS have been associated with increased risk for cardiac arrhythmias.

OBJECTIVES: To describe the prevalence and correlates among Nigerians with heart failure.

METHODS: A total of 129 subjects were consecutively recruited for the present study. QT interval, QRS duration and fQRS were determined. SPSS version 16 was used for analysis.

RESULTS: The mean $( \pm \mathrm{SD})$ age was $62.1 \pm 13.7$ years $(43.4 \%$ female). Mean QT, QTc and QRS durations were $390.4 \pm 56.2 \mathrm{~ms}, 451.3 \pm 62.7 \mathrm{~ms}$ and

Cragmented QRS (fQRS), an easily evaluated noninvasive electrocardiographic parameter, is an important marker of myocardial injury (1). It is defined as additional spikes within the QRS complex; or the presence of an additional $\mathrm{R}$ wave $\left(\mathrm{R}^{\prime}\right)$; or notching in the nadir of the $S$ wave; or the presence of $>1 R^{\prime}$ in two contiguous leads corresponding to a major coronary artery territory on the resting 12-lead electrocardiogram (ECG) (Figure 1). fQRS has been associated with, and is also a predictor of, mortality and arrhythmic events in patients with reduced left ventricular systolic function among Caucasians $(2,3)$. Although fQRS was initially used as a marker of myocardial scar in coronary artery disease, its usefulness has been expanded to other diseases such as cardiac sarcoidosis, arrhythmogenic right ventricular cardiomyopathy, hypertrophic cardiomyopathy, Brugada syndrome and acquired long QT syndrome $(4,5)$. fQRS complex has also been proposed as an additional tool to separate individuals with left ventricular diastolic dysfunction from those with heart failure with preserved ejection fraction (6).

In a review of 50 cases of sudden death from Ile-Ife (southwestern Nigeria), death was attributed to acute left ventricular failure in $68 \%$ of the subjects, while only $4 \%$ of deaths were attributed to acute myocardial infarction (7). In a recent nine-year review of cases of sudden death from Osogbo, Nigeria, cardiovascular disease, especially heart failure, was the most common cause of sudden death (8). In another review of autopsied cases in southern Nigeria over a nine-year period, cardiovascular disease was the most common cause of sudden death, accounting for $87.6 \%$ of all-cause mortality (9).

Sudden cardiac death is the most common cause of mortality in heart failure subjects (6), which is often related to malignant supraventricular and ventricular arrhythmias (10). Conventional markers, such as left ventricular ejection fraction, have been widely used to predict mortality $(10,11)$. The initiation and maintenance of re-entrant ventricular arrhythmias depends on the triggers and a vulnerable myocardial substrate (12). Depolarization and repolarization abnormalities including fQRS, microwave T wave alternans, QT prolongation, QT dispersion
99.5 \pm 23.9 ms, respectively. fQRS, QTc prolongation and wide QRS occurred in $41.1 \%, 44.2 \%$ and $41.9 \%$, respectively, of study participants. fQRS appeared to be more likely associated with prolonged QTc, increased heart rate, presence of old myocardial infarction and wide QRS duration among study participants.

CONCLUSION: fQRS, QTc prolongation and wide QRS are frequent among Nigerians with heart failure. Appropriate screening for heart failure is suggested for early intervention.

Key Words: Clinical correlates; Electrocardiographic abnormalities; Fragmented QRS; Heart failure; Prevalence

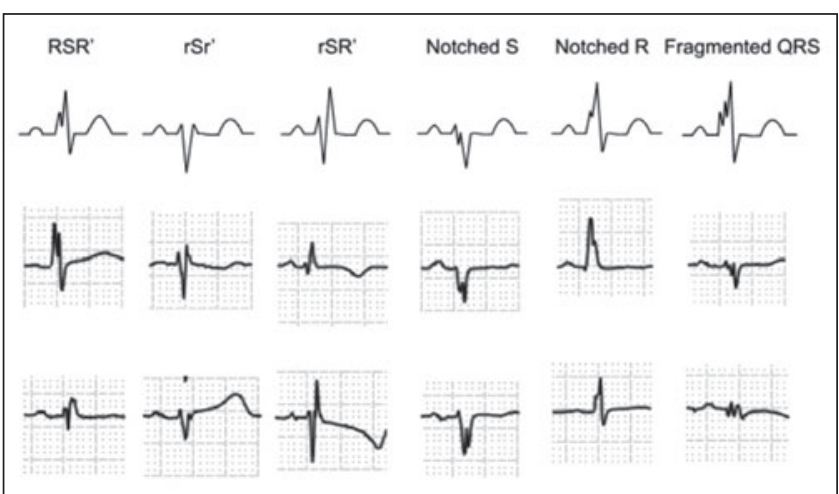

Figure 1) Example of fragmented QRS pattern

and wide QRS have been shown to predict sudden cardiac death in many populations $(1,8,12)$. Das and Masry (12) demonstrated that the fQRS represents conduction delay due to myocardial scar in patients with coronary artery disease, and that it is also similar for other myocardial diseases. fQRS are, therefore, potential substrates for initiation of cardiac arrhythmias in patients with heart failure. While this has been studied among the Caucasian population, reports of fQRS and other depolarization abnormalities are rare among Nigerians.

Accordingly, we aimed to determine the frequency of $\mathrm{fQRS}$ and QT prolongation among a group of Nigerian subjects with heart failure, and describe the clinical and demographic characteristics as well as associated clinical correlates.

\section{METHODS}

The study was performed in the Goshen Heart Clinic, Osogbo, Nigeria. The clinical records of all heart failure cases seen between May 2011 and December 2014 were retrieved. All potential subjects were included if they were $>18$ years of age. Subjects with serious

${ }^{1}$ Department of Medicine, Ladoke Akintola University of Technology (LAUTECH) E⿱ LAUTECH Teaching Hospital, Ogbomoso; ${ }^{2}$ Goshen Heart Clinic, Osogbo, Nigeria

Correspondence: Dr AA Akintunde, PO Box 3238, Osogbo, Osun State, Nigeria. Telephone 234-803-393-2076,

e-mail: iakintunde2@yahoo.com, aaakintunde@lautech.edu.ng 
TABLE 1

The clinical characteristics of heart failure subjects

\begin{tabular}{lc}
\hline Variable & Results \\
\hline Age, years & $62.1 \pm 13.7$ \\
Female sex, $\mathrm{n}(\%)$ & $56(43.4)$ \\
Systolic blood pressure, $\mathrm{mmHg}$ & $136.6 \pm 28.6$ \\
Diastolic blood pressure, $\mathrm{mmHg}$ & $83.2 \pm 17.6$ \\
Fasting blood sugar, mmol/L & $4.7 \pm 2.2$ \\
Left ventricular internal dimension in diastole, mm & $49.0 \pm 11.9$ \\
Left ventricular end-systolic dimension, mm & $37.3 \pm 12.7$ \\
Posterior wall thickness in diastole, mm & $12.6 \pm 1.9$ \\
Interventricular septal thickness, mm & $12.7 \pm 2.4$ \\
Mean mitral E/A ratio & $1.1 \pm 0.8$ \\
Mean tricuspid E/A ratio & $1.1 \pm 0.7$ \\
Ejection fraction, \% & $56.2 \pm 12.4$ \\
Right ventricular dimension, mm & $29.5 \pm 5.4$ \\
Left atrial dimension, mm & $45.9 \pm 9.2$ \\
Relative wall motion abnormalities, $\mathrm{n}(\%)$ & $8(6.2)$ \\
ST-T wave abnormalities, $\mathrm{n}$ (\%) & $68(52.7)$ \\
Pathological Q wave in contiguous leads, $\mathrm{n}(\%)$ & $13(10.1)$ \\
Proportion of those with possible silent old coronary & $18 / 129(13.95)$ \\
artery disease, $\mathrm{n} / \mathrm{n}$ (\%) & \\
Prevalence of fragmented QRS, $\mathrm{n}(\%)$ & $53(41.1)$ \\
QT duration, ms & $390.4 \pm 56.2$ \\
QTc prolongation, $\mathrm{n}$ (\%) & $57(44.18)$ \\
Wide QRS (>100 ms), $\mathrm{n}$ (\%) & $54(41.9)$ \\
Heart rate, beats/min & $79.5 \pm 19.6$ \\
QRS duration, ms & $99.5 \pm 23.9$ \\
QTc duration, ms & $451.3 \pm 62.7$ \\
New York Heart Association III/IV, $\mathrm{n}(\%)$ & $41(31.7)$ \\
Heart failure with preserved ejection fraction, $\mathrm{n}(\%)$ & $67(51.9)$ \\
\hline Data present as mean \pm SD uns &
\end{tabular}

Data presented as mean $\pm S D$ unless otherwise indicated

comorbidities including cancers, advanced kidney disease and stroke were excluded. Subjects with metabolic abnormalities, such as hyperkalemia, those taking tricyclic antidepressants, early repolarization abnormalities and history suggestive of pulmonary embolism, were also excluded from the analysis. Information obtained from the clinical records included age, sex, occupation, clinical features of heart failure, drug history, history of hypertension and diabetes mellitus, smoking history, alcohol history and duration of symptoms. Height, weight, waist circumference, average systolic and diastolic blood pressure, and heart rate were obtained. Electrocardiography was performed using the ECG 1200 (Contec Medical Systems, China). Echocardiography was performed using the HP Sonos 2500 (HP Inc, USA) with a $2.7 / 3.5 \mathrm{MHz}$ probe. Echocardiography was performed according to standardized American Society of Echocardiography guidelines on quantification and evaluation of systolic and diastolic parameters and chambers assessment (13). The following parameters were obtained: left ventricular internal dimension in diastole, left ventricular internal dimension in systole, left ventricular posterior and septal wall dimension in diastole, ejection fraction, fractional shortening, left atrial dimension, right ventricular wall dimension and aortic root dimension. Global and regional assessment for wall motion abnormalities were made visually and reported. The electrocardiography was interpreted by the author blinded to the clinical data of the subjects. Parameters such as heart rate, rhythm, QRS axis, PR interval and QTc were obtained. Left ventricular hypertrophy was defined using the Sokolow-Lyon criteria $(11,13)$. Fasting blood sugar, lipid profile including high-density lipoprotein cholesterol, lowdensity lipoprotein cholesterol, total cholesterol and triglycerides were obtained using a rapid point-of-care, strip-based test (LipidPro; Infopia Ltd, Korea).
TABLE 2

Clinical and electrocardiographic differences between those with and without fragmented QRS (fQRS) on 12-lead electrocardiogram

\begin{tabular}{lrcc}
\hline \multirow{2}{*}{ Variable } & \multicolumn{2}{c}{ fQRS } & \\
\cline { 2 - 3 } Heart rate, beats/min & Present & Absent & P \\
QRS duration, ms & $87.2 \pm 18.3$ & $80.4 \pm 17.3$ & $0.047^{*}$ \\
QT duration, ms & $100.2 \pm 25.0$ & $96.6 \pm 22.0$ & 0.520 \\
QTc, ms & $391.4 \pm 58.5$ & $389.0 \pm 48.6$ & 0.979 \\
Age, years & $463.9 \pm 70.3$ & $448.3 \pm 46.3$ & 0.698 \\
Female sex, $\mathrm{n}(\%)$ & $60.8 \pm 15.8$ & $62.2 \pm 12.2$ & 0.894 \\
Prolonged QTc, $\mathrm{n}$ & $20(15.5)$ & $37(28.7)$ & 0.610 \\
Old myocardial infarction, $\mathrm{n}$ & 32 & 21 & $0.000^{\star}$ \\
Wide QRS, n & 11 & 7 & $0.002^{*}$ \\
Ejection fraction, \% & 39 & 13 & $0.000^{*}$ \\
\hline
\end{tabular}

Data presented as mean $\pm S D$ unless otherwise indicated. * ${ }^{*}$ tatistically significant

fQRS was defined on the 12-lead ECG including various morphologies of the QRS wave with or without a $Q$ wave. fQRS include the presence of an additional $\mathrm{R}$ wave $\left(\mathrm{R}^{\prime}\right)$ or notching in the nadir of the $\mathrm{R}$ wave or the $\mathrm{S}$ wave, or the presence of $>1 \mathrm{R}^{\prime}$ (fragmentation) in two contiguous leads corresponding to a major coronary artery territory. In the presence of left or right bundle branch block or premature ventricular complexes, fQRS was defined as the presence of $>2$ notches in the $\mathrm{R}$ or $\mathrm{S}$ wave $(1,2,10)$.

QT intervals including the QT duration and QTc duration were calculated using ECG Synchronous V1.3.3 computer software (Contec Medical Systems, China). Prolonged QTc was defined as QTc $>460 \mathrm{~ms}$ in women and $>440 \mathrm{~ms}$ in men. Wide QRS was defined as QRS duration $>100 \mathrm{~ms}$ (6,12). Data were analyzed using SPSS version 16.0 (IBM Corporation, USA). Numerical data were summarized as mean \pm SD. Qualitative data were summarized as frequency and percentages. Student's $t$ test and the $\chi^{2}$ test were used as appropriate to determine differences between groups; $\mathrm{P}<0.05$ was considered to be statistically significant.

\section{RESULTS}

Table 1 shows the clinical characteristics of heart failure subjects. The mean age was $62.1 \pm 13.7$ years. There were $56(43.4 \%)$ women in the study group. The mean systolic and diastolic blood pressures were $136.6 \pm 28.6 \mathrm{mmHg}$ and $83.2 \pm 17.6 \mathrm{mmHg}$, respectively, while the mean fasting blood sugar was $4.7 \pm 2.2 \mathrm{mmol} / \mathrm{L}$. The mean ejection fraction was $56.2 \pm 12.4 \%$. fQRS was documented in $53(41.1 \%)$ of heart failure patients. QTc prolongation and wide QRS were found in $57(44.2 \%)$ and $54(41.9 \%)$, respectively. The mean heart rate was $79.5 \pm 19.6$ beats $/ \mathrm{min}$ while the mean QRS duration was $99.5 \pm 23.9 \mathrm{~ms}$. Mean QTc duration was $451.3 \pm 62.7$ ms. Relative wall abnormalities seen on echocardiography was demonstrated among eight (6.2\%) study participants. ST-T wave abnormalities were more common, and were evident in $68(52.7 \%)$ of heart failure subjects in the present study. Pathological Q wave was found in 13 (10.1\%) of study participants while evidence of old myocardial infarction was found in $18(13.95 \%)$. Most patients had left atrial dilation. The mean left atrial dimension was $45.9 \pm 9.2 \mathrm{~mm}$.

The clinical, demographic and electrocardiographic differences between those with fQRS and those without fQRS are shown in Table 2. Those with $\mathrm{fQRS}$ were more likely to have a significantly higher heart rate compared with those without fQRS $(87.2 \pm 18.3$ beats/min versus $80.4 \pm 17.3$ beats/min; $\mathrm{P}<0.05)$. Although mean QRS duration and mean QTc duration were higher among those with fQRS, the differences were not statistically significant. However, the proportion of those with prolonged QTc, old myocardial infarction and wide QRS duration were significantly higher among those with fQRS compared with those 
without $\mathrm{fQRS}$ in the present study population. Mean ejection fraction was significantly lower among subjects with $\mathrm{fQRS}$ compared with those without $(46.1 \pm 11.0 \%$ versus $58.3 \pm 12.7 \%$; $\mathrm{P}<0.05)$.

\section{DISCUSSION}

Heart failure is fast becoming a major cause of increasing morbidity and mortality in Africa. This is due to the increasing prevalence of its many associated risk factors including hypertension, diabetes, etc; availability of newer drugs; and the influence of technology in management such as availability of pacemakers, implantable cardioverter defibrillators, etc. In heart failure, the prognosis has been likened to that of a malignancy. The most common cause of death in heart failure is due to sudden cardiac death, which is linked to arrhythmias (6,8-9). It is pertinent to identify potential markers of susceptibility to increased cardiac death among Africans (1,3). fQRS has been shown to be a major marker of myocardial scar/injury in coronary artery disease and many other diseases with high sensitivity and specificity (14).

The present study revealed that fQRS is common among Nigerians with heart failure because it occurred in $41.4 \%$ of study participants. This is similar to reports from other parts of the world. In a study by Rad et al (15), 41.5\% of heart failure patients in Iran were reported to have fQRS. In that study, they also showed that $\mathrm{fQRS}$ is a sign of myocardial scar and predicts nonresponsiveness to cardiac resynchronization therapy in heart failure, a recent technologically derived, device-based therapy useful in biventricular noncompaction and impaired synchronization and advanced heart failure. The presence of $\mathrm{fQRS}$ has been shown to be associated with a significantly higher all-cause mortality even after adjusting for other conventional risk markers such as ejection fraction, age, history of diabetes mellitus, etc (16). This was also compatible with our findings in the present study, in which subjects with fQRS were more likely to have increased heart rate, lower ejection fraction and other depolarization abnormalities compared with those without fQRS. In other reports from other developing countries such as India and China, fQRS was reported in $23 \%$ to $75 \%$ of patients with dilated cardiomyopathy and heart failure, and it was associated with interventricular dysynchrony $(16,17)$.

The present study also revealed that a significant proportion of heart failure patients had prolonged QTc and wide QRS (QRS duration $>100 \mathrm{~ms}$ ) and that they were closely linked to the presence of fQRS and other potential markers of increased mortality such as increased age and the association with the presence of ECG changes suggestive of old myocardial infarction. This is also similar to other reports from around the world. Kashani and Barold (18) reported that prolonged QRS (QRS duration >120 ms) occurs in 14\% to $47 \%$ of patients with heart failure. QRS prolongation and wide QRS have been shown to be significant predictors of left ventricular systolic dysfunction in patients with heart failure and are, consequently, predictors of poor prognosis in heart failure $(18,19)$. Heart failure patients with QRS prolongation and wide QRS have higher all-cause mortality and, possibly, a higher incidence of sudden death, and this increases as the QRS duration increases (20).

QT and QTc prolongation is also associated with increased risk for cardiovascular death $(6,19)$. The QT interval is a measure of the duration of ventricular depolarization and repolarization, and is a method of screening for those at increased risk of cardiac arrhythmias (21). QTc prolongation is also associated with various components of the insulin resistance syndrome, age, body mass index, left ventricular hypertrophy and resistant hypertension (22). A significant proportion of study participants also had QTc prolongation and this was more frequent among women than men. Prolonged QTc was also associated with the presence of $\mathrm{fQRS}$ among the study participants. This is similar to what was reported in other studies from other part of the world. Sandhu and Bahler (19) reported QTc prolongation in $43 \%$ of heart failure patients undergoing surgical revascularization and also concluded that patients with heart failure and preoperative QTc interval prolongation have increased mortality rates after coronary artery bypass grafting.
The proportion of QTc prolongation found in this study is, however, less than what was described by Kolo et al $(23,24)$ from North Central Nigeria among chronic heart failure patients; in that study, $63 \%$ of subjects had QTc prolongation. We suggest that those patients possibly had a more advanced heart failure as evidenced by their mean ejection fraction, which was significantly lower than that in our study. Arrhythmias are not uncommon in Nigerians with heart failure; $24 \mathrm{~h}$ ambulatory ECG monitoring has shown that ambulant outpatients with chronic heart failure have significantly higher prevalence of ventricular arrhythmias and narrow heart rate variability compared with healthy individuals of comparable age and sex $(23)$. Kolo et al $(23,24)$ also showed that QTc is an important predictor of mortality in subjects with chronic heart failure in Nigeria. The presence of fQRS represents distortion of signal conduction and depolarization process within the ventricles and is related to myocardial scar/ischemia/fibrosis. It is not surprising that $\mathrm{fQRS}$ is related to QT prolongation and QRS duration $>100 \mathrm{~ms}$ in the present study, which are widely acceptable conventional markers of increased cardiac susceptibility to cardiac arrhythmias and cardiovascular death in the general population and, specifically, in heart failure patients $(9,10,18,20)$. Although there is controversial evidence on its specificity as a screening tool for proarrhythmias, it still has significant prognostic and economic value in many cardiac and noncardiac diseases. Other causes of QT prolongation include medications such as beta-blockers, digoxin, etc, which most of our heart failure patients were using. Other causes of QT abnormalities including congenital long QT syndrome. Treatments for these abnormalities are multidimensional, and includes appropriate evaluation and monitoring, the withdrawal of offensive medications, use of implantable cardioverter defibrillators, surgical revascularization and correction of electrolyte imbalance, etc.

\section{CONCLUSION}

fQRS, QTc prolongation and wide QRS duration frequently occur among Nigerians with heart failure and may be potential predictors of increased cardiovascular susceptibility to cardiac arrhythmias and sudden cardiac death among heart failure patients. Monitoring is, therefore, essential. Appropriate interventions including withdrawal of some medications, use of devices such as implantable cardioverter defibrillators, among others, after appropriate criteria have been met, are necessary to reduce the increased risk of cardiac death among these patients. We therefore suggest routine measurement of QT parameters and identifying fQRS to properly identify, follow up and manage heart failure subjects with high cardiovascular risk.

DISCLOSURES: The authors have no financial disclosures or conflicts of interest to declare.

\section{REFERENCES}

1. Das MK, Khan B, Jacob S, et al. Significance of a fragmented QRS complex versus a $Q$ wave in patients with coronary artery disease. Circulation 2006;113:2495-501.

2. Das MK, Zipes DP. Fragmented QRS: A predictor of mortality and sudden cardiac death. Heart Rhythm 2009;6:S8-14.

3. Basaran Y, Tigen K, Karaahmet T, et al. Fragmented QRS complexes are associated with cardiac fibrosis and significant intraventricular systolic dyssynchrony in non-ischemic dilated cardiomyopathy patients with a narrow QRS interval.

Echocardiography 2011;28:62-8.

4. Homsi M, Alsayed L, Safadi B, Mahenthiran J, Das MK. Fragmented QRS complexes on 12-lead ECG: A marker of cardiac sarcoidosis as detected by gadolinium cardiac magnetic resonance imaging. Ann Noninvasive Electrocardiol 2009;14:319-26.

5. Park SJ, On YK, Kim JS, et al. Relation of fragmented QRS complex to right ventricular fibrosis detected by late gadolinium enhancement cardiac magnetic resonance in adults with repaired Tetralogy of Fallot. Am J Cardiol 2012;109:110-5.

6. Gomes JA, Cain ME, Buxton AE, Josephson ME, Lee KL, Hafley GE. Prediction of long-term outcomes by signal-averaged electrocardiography in patients with unsustained ventricular 
tachycardia, coronary artery disease, and left ventricular dysfunction. Circulation 2001;104:436-41.

7. Rotimi O, Ajayi AA, Odesanmi WO. Sudden unexpected death from cardiac causes in Nigerians: A review of 50 autopsied cases. Int J Cardiol 1998;63:111-5.

8. Akinwusi PO, Komolafe AO, Olayemi OO, Adeomi AA. Pattern of sudden death at Ladoke Akintola University of Technology Teaching Hospital, Osogbo, South West Nigeria. Vasc Health Risk Manag 2013;9:333-9.

9. Obiora CC, Amakiri CN. Systemic analysis of sudden natural deaths at Braithwaite Specialist Hospital, Port Harcourt, Nigeria. Nigerian Health J 2012;12:47-51.

10. Onoue Y, Izumiya Y, Hanatani S, et al. Fragmented QRS complex is a diagnostic tool in patients with left ventricular diastolic dysfunction. Heart Vessels 2015 Feb 25 [Epub ahead of print]. PMID 25712607.

11. Jern S. Assessment of left ventricular hypertrophy in patients with essential hypertension. Blood Press Suppl 1997;2:16-23.

12. Das MK, Masry HE. Fragmented QRS and other depolarization abnormalities as a predictor of mortality and sudden cardiac death. Curr Opin Cardiol 2010;25:59-64.

13. Lang RM, Badano LP, Mor-Avi V, et al. Recommendation for cardiac chamber quantification by echocardiography in adults: An update from the American Society of Echocardiography and European Society of Cardiovascular Imaging. J Am Soc Echocardiogr 2015;28:1-39.

14. Ari H, Centinkaya S, Ari S, Koca V, Bozat T. The prognostic significance of a fragmented QRS complex after primary percutaneous coronary intervention. Heart Vessels 2012;27:20-8.

15. Rad MA, Baboli NT, Barzigar A, et al. The role of fragmented QRS complexes on a routine 12 lead ECG in predicting non responsiveness to cardiac resynchronization therapy. Anat J Cardiol 2015;15:204-8.
16. Das MK, Suradi H, Maskoun W, et al. Fragmented wide QRS on a 12-lead ECG: A sign of myocardial scar and poor prognosis. Circ Arrhythm Electrophysiol 2008;1:258-68.

17. Take Y, Morita H. Fragmented QRS: What is the meaning? Indian Pacing Electrophysiol J 2012;12:213-25.

18. Kashani A, Barold SS. Significance of QRS complex duration in patients with heart failure. J Am Coll Cardiol 2005;46:2183-92.

19. Sandhu R, Bahler RC. Prevalence of QRS prolongation in a community hospital cohort of patients with heart failure and its relation to left ventricular systolic dysfunction. Am J Cardiol 2004;93:244-6.

20. Kalra PR, Sharma R, Shamin W, et al. Clinical characteristics and survival of patients with chronic heart failure and prolonged QRS duration. Int J Cardiol 2002;86:225-31.

21. Saadeh A, Evans S, James M, Jones J. QTc dispersion and complex ventricular arrhythmias in untreated newly presenting hypertensive patients. J Hum Hypertens 1999;13:665-9.

22. Akintunde AA, Oyedeji AT, Familoni OB, Ayodele OE, Opadijo OG. QT interval prolongation and dispersion: Epidemiology and clinical correlates in subjects with newly diagnosed systemic hypertension in Nigeria. J Cardiovasc Dis Res 2012;3:290-5.

23. Kolo PM, Opadijo OG, Omotoso AB, Balogun MO, Araoye MA, Katibi IA. Prevalence of QTc prolongation in adult Nigerians with chronic heart failure. West Afr J Med 2008;27:69-73.

24. Kolo PM, Opadijo OG, Omotoso AB, Katibi IA, Balogun MO, Araoye MA. Prognostic significance of QT interval prolongation in adult Nigerians with chronic heart failure. Nig J Clin Pract 2008;11:336-41. 